\title{
CONSTRUCCIÓN DE MAPAS DE RUIDO CON ELEMENTOS FINITOS Y NEWMARK
}

\section{CONSTRUCTION NOISE MAPS WITH FINITE ELEMENT AND NEWMARK}

\author{
Irla Mantilla ${ }^{1}$, Jonathan Munguia ${ }^{2}$ \\ RESUMEN
}

En el presente trabajo se construye un modelo matemático para la construcción de Mapas de Ruido basado en Ecuaciones Variacionales Hiperbólicas (EVP), el cual, se obtiene de la formulación débil del problema de Contorno y Condiciones iniciales de Cauchy asociadas a Ecuaciones Diferenciales en Derivadas Parciales de tipo Hiperbólico. Para garantizar la simulación numérica del problema de propagación de la fuente de ruido se obtiene su formulación variacional en espacios de tipo Sobolev evolutivos, así se prueba la existencia y unicidad de solución del problema variacional, luego para resolver el problema se aplica el método de Galerkin con Elementos Finitos para la discretización espacial y el método de Newmark para la discretización temporal. En este trabajo se innova la técnica de preparación de la base de datos y la experimentación computacional con Matlab, obteniendo finalmente eficazmente la solución como se muestra en la convergencia del esquema numérico.

Palabras clave.- Mapas de ruido, Método de Galerkin, Elementos finitos, Método de Newmark.

\begin{abstract}
This article uses the weak formulation of the problems Partial Hyperbolic type (VPE) for construction noise maps with finite element and Newmark in two-dimensional space. To ensure the numerical simulation of the problem of propagation of the noise source, so that proves the existence and uniqueness of the variational problem in Sobolev spaces evolutionary and applied the finite element method and method Galerkin for spatial discretization and Newmark method for the time discretization. In this work, the innovative technique of preparation of the data base and computational experimentation whit Mathlab, finally obtaining effectively the solution as shown in the convergence of the numerical scheme.
\end{abstract}

Key words.- Noise maps, Galerkin method, Finite elements, Newmark method.

\section{INTRODUCCIÓN}

Para el control del nivel de contaminación acústica en el Perú, se ha trazado algunas estrategias para la medición de ruidos tales como la colocación de sonómetros en lugares específicos asociados a fuentes de ruido. La construcción de un Mapa de
Ruido desde este punto de vista es muy costosa y es el máximo indicador [1] donde se muestra los diferentes niveles sonoros. En un informe sobre estudios acústicos, manifiestan que una exhaustiva medición mediante el uso de Sonómetros y su innovación en cuanto a su medición, es un tema actual de investigación.

\footnotetext{
${ }^{1}$ Dra. Docente investigadora de la Facultad de Ciencias de la Universidad Nacional de Ingeniería, ${ }^{2}$ Docente investigador de la Facultad de Ciencias de la Universidad Nacional de Ingeniería.
} 
La importancia de una buena medición es fundamental, ya que cualquier error puede propagarse en la calidad del mapeo.

Generalmente se estudia la repercusión de las fuentes de ruido en modo experimental aplicando formulaciones empíricas. De esta naturaleza existen muchos estudios pero no son suficientes para la construcción de un mapeo de calidad.

En el presente trabajo se pretende abordar la construcción de un mapa de ruido mostrando otra alternativa la cual comprende la construcción de un modelo matemático basado en un problema de contorno y condición inicial asociado a una ecuación diferencial en derivadas parciales hiperbólicas de segundo orden para la variable presión sonora (PS), el cual, se transforma en una ecuación variacional hiperbólica $(\mathrm{EVH})$ definida sobre un subconjunto de $\mathbb{R}^{n}$, para $\mathrm{n}=3$, y se asume sobre un conjunto de puntos expuesto a diferentes fuentes sonoras.

El modelo será capaz de simular el comportamiento de la presión sonora sobre dicho conjunto.

\section{MODELO MATEMÁTICO}

Para nuestros fines se considera un flujo de aire sobre un conjunto $\Omega_{T} \subset \mathbb{R}^{n+1}$, para $n=2$ que representa el dominio evolutivo y espacialmente está determinado sobre un sistema de coordenadas $\{X, Z\}$ y temporalmente sobre un intervalo $[0, \mathrm{~T}), \mathrm{T}>0$.

Sean $\rho=\rho(x, z, t)$, la densidad del aire, $p=$ $p(x, z, t)$ la presión sonora (PS) y las fuerzas externas representadas por la función $F=$ $F(x, z, t)$ calculadas para una unidad de masa de aire, en el punto $(x, z)$ y en un instante $t$. Además $v=\left(v_{x}, v_{z}, v_{t}\right)$ es la velocidad de la partícula de aire, donde $v_{x}, v_{z}$ son las componentes en las direcciones $X, Z$.

Estas magnitudes caracterizan la perturbación del aire, generada por la presión de dicha fuente de ruido. El comportamiento fenomenológico [2] está gobernado por las siguientes ecuaciones de conservación: a) Ecuación de cantidad de movimiento.-

$$
v_{t}+v \cdot \nabla v=-\frac{1}{\rho} \nabla p+F
$$

b) Ecuación de continuidad.-

$$
\rho_{t}+v \cdot \nabla \rho+\rho \nabla \cdot \mathrm{v}=0
$$

En nuestro caso se asume que no actúan las fuerzas externas sobre la propagación del ruido, es decir, $F=0$, y que dicho proceso es adiabático, entonces, las oscilaciones del aire son pequeñas, de este modo se puede simplificar el modelo haciendo:

$$
\begin{aligned}
& v_{t}=-\frac{1}{\rho_{0}} \nabla p, \\
& \rho_{t}+\rho_{0} \nabla \cdot v=0, \\
& p=c^{2} \rho
\end{aligned}
$$

En el que se ha despreciado los términos no lineales de las ecuaciones de conservación, bajo la suposición de pequeñas oscilaciones y considerando que $c=\sqrt{\frac{\gamma p_{0}}{\rho_{0}}}$ es la velocidad del sonido, se obtiene de la suposición adiabática, que ésta según el Sistema Internacional (SI) mide aproximadamente $343[\mathrm{~m} / \mathrm{s}]$.

Para $t=t_{0}$, sean $p_{0}$ la presión inicial, $\rho_{0}$ la densidad inicial, $\gamma$ un parámetro que estima el cociente del calor específico a presión constante entre el calor específico a volumen constante [1].

Combinando las ecuaciones (1), (2) y (3), se obtiene la siguiente ecuación:

$$
\frac{\partial^{2} p}{\partial t^{2}}=c^{2} \Delta p
$$

Estas ecuaciones pertenecen al conjunto de ecuaciones diferenciales en derivadas parciales del tipo hiperbólico (EDPH).

En general un problema EDPH con valores iniciales $\mathrm{y}$ de contorno se expresa mediante el siguiente sistema de ecuaciones: 
$(P D)\left\{\begin{array}{c}\frac{\partial^{2} p}{\partial t^{2}}+L[p]=f \text { sobre }_{T} \\ p=g, \quad p_{t}=h \text { sobre } \Omega \times\{t=0\} \\ p=0 \text { sobre } \Omega \times[0, T]\end{array}\right.$

Donde: las funciones $f, g$ y $h$ son continuas y están definidaspor $f: \Omega_{T} \subset \mathbb{R}^{n+1} \rightarrow \mathbb{R}, g, h: \Omega \subset \mathbb{R}^{n} \rightarrow$ $\mathbb{R}$ y $p: \bar{\Omega}_{T} \subset \mathbb{R}^{n+1} \rightarrow \mathbb{R}$ es la función a determinar, $\Omega$ es un subconjunto de puntos en $\mathbb{R}^{n}$ donde se evaluará la presión sonora (PS), y $\Omega_{T}=\Omega \times[0, T]$ es un subconjunto de puntos en $\mathbb{R}^{n+1}$, donde se analiza la PS evolutiva.

El operador diferencial $L$, lineal de segundo orden es expresado por:

$$
\begin{gathered}
L[p]=-\sum_{i, j=1}^{n}\left(a_{i j}(x, t) p_{x_{i}}\right)_{x_{j}}+\sum_{i=1}^{n} b_{i}(x, t) p_{x_{i}} \\
+c(x, \mathrm{t}) p
\end{gathered}
$$

cuyos coeficientes $a_{i j}, b_{i}, c$ son funciones continuas y dependen de $(x, t) \in \Omega_{T}$.

Según [2], si existe una constante $\theta>0$, tal que:

$$
\begin{gathered}
\forall(x, t) \in \Omega_{T}, \quad \forall y \in \mathbb{R}^{n} \text { se cumple } \\
\sum_{i, j=1}^{n} a_{i j}(x, t) y_{i} y_{j} \geq \theta|y|^{2} .
\end{gathered}
$$

Entonces se define al operador $\frac{\partial^{2}}{\partial t^{2}}+L$ de la ecuación (5a) como un operador hiperbólico uniforme.

El operador diferencial de la ecuación (4) en particular es hiperbólico uniforme con $a_{i j}(x, t)=$ $c^{2}$.

\section{RESOLUCIÓN DEL PROBLEMA EDPH}

La solución aproximada de este problema se encuentra en un subespacio de $V$, donde

$$
V=\left\{\begin{array}{c}
p \in C^{2}(\Omega) \cap C\left(\overline{\Omega)}: \forall(x, t) \in \Omega_{T},\right. \\
p(x, 0)=g, \quad \partial_{t} p(x, 0)=h, \\
p=0 \text { sobre } \partial \Omega \times[0, T]
\end{array}\right\}
$$

Para ello se debe determinar la formulación variacional, en este caso se aplica el teorema de la divergencia en un espacio de Hilbert del tipo Sobolev evolutivo [3].

Fijando $x$ se redefine a $p$ y $f$, como:

$$
\bar{p}:[0, T] \rightarrow H_{0}^{1}\left(\varsigma_{1}\right) \text { por }[\bar{p}(t)](x):=p(x, t),
$$

de manera similar

$$
\bar{f}:[0, T] \rightarrow L^{2}(\Omega) \text { por }[\bar{f}(t)](x):=f(x, t) .
$$

Considerando la siguiente notación, para la regularidad de las variables evolutivas en tiempo:

$\bar{p} \in L^{2}\left(0, T ; H_{0}^{1}\left(S_{1}\right)\right)$, representa a la primera derivada por $\bar{p}^{\prime} \in L^{2}\left(0, T ; L^{2}(\Omega)\right)$ y a la segunda derivada parcial respecto al tiempo por $\bar{p}^{\prime \prime} \in$ $L^{2}\left(0, T ; H^{-1}(\Omega)\right)$, donde $H_{0}^{1}\left(\Omega_{\text {. }}\right)$ es un subespacio de $V$ y $H^{-1}(\Omega)$ es el espacio dual de $H_{0}^{1}\left(\Omega_{1}\right)$.

Sea $L^{2}\left(0, T ; L^{2}(\Omega)\right)$ el subespacio evolutivo de clases de equivalencia [4] de tal modo que multiplicando por una función de prueba $v \in H_{0}^{1}\left(\Omega_{1}\right)$ e integrando sobre la clausura de $\Omega$ del problema (PD), éste se convierte en las ecuaciones (6) y (7):

$$
\left\langle\bar{p}^{\prime \prime}, \boldsymbol{v}\right\rangle+B[\bar{p}, \boldsymbol{v} ; t]=(\bar{F}, \boldsymbol{v})
$$

para todo $v \in H_{0}^{1}\left(\Omega_{1}\right)$, en $t \in[0, T]$ y que cumple las condiciones iniciales:

$$
\bar{p}(0)=g \quad \text { y } \quad \bar{p}^{\prime}(0)=h
$$

A las ecuaciones (6) y (7) se denomina problema variacional hiperbólico (PVH), (,) denota el producto interno de $L^{2}(\Omega),\langle$,$\rangle denota al producto de$ dualidad entre $H^{-1}(\Omega)$ y $H_{0}^{1}(\Omega)$ y $B[., . ;$.$] es una$ forma bilineal evolutiva, definida por: 


$$
\begin{gathered}
B\left[\bar{p}, \boldsymbol{v}_{j} t\right]=\int_{\Omega} \sum_{i, j=1}^{n} a_{i j}(., t) \bar{p}_{x_{i}} \boldsymbol{v}_{x_{j}} \\
+\int_{\Omega} \sum_{i=1}^{n} b_{i}(., t) \bar{p}_{x_{i}} \boldsymbol{v} \\
+c(., \mathrm{t}) \bar{p} \boldsymbol{v} d x \\
\int_{\Omega}\left[\frac{1}{c^{2}} \boldsymbol{v} \frac{\partial^{2} \bar{p}}{\partial t^{2}}+\frac{\partial v}{\partial x} \frac{\partial \bar{p}}{\partial x}+\frac{\partial v}{\partial z} \frac{\partial \bar{p}}{\partial z}\right] d x d z \\
=0
\end{gathered}
$$

Se demuestra en [3] que tiene solución única.

\section{RESOLUCIÓN NUMÉRICA}

Sea una sucesión de funciones $\left\{\psi_{k}\right\}_{k=1}^{\infty}$, que satisfacen las condiciones de regularidad tal que define una base ortogonal de $H_{0}^{1}(\Omega)$ y una base ortonormal de $L^{2}(\Omega)$. Por el teorema fundamental del algebra toda base infinita contiene una base finita del subespacio aproximador, fijando $m$ entero positivo, y aplicando el método de Galerkin, se puede definir como base del subespacio de dimensión finita $m$, la siguiente igualdad:

$$
\bar{p}_{m}:=\sum_{k=1}^{m}\left(g, \psi_{k}\right) \psi_{k}
$$

En $0 \leq t \leq T, \quad$ y $\quad k=1, \ldots, m$, se asume que $\bar{p}_{n}$ satisface la siguiente ecuación variacional.

$$
\left\langle\bar{p}_{n}{ }^{\prime \prime}, \psi_{k}\right\rangle+B\left[\bar{p}_{m}, \psi_{k} ; t\right]=0
$$

Entonces resulta que el problema diferencial de la presión donora expresada por la ecuación (4) y (8) tiene como solución $\bar{p}_{n}$ de la ecuación variacional hiperbólica discreta. Ahora nuestro problema es encontrar la resolución de la ecuación (10) en la que se utiliza el método Galerkin descrito en (9) y (10) elementos finitos con funciones de forma polinomial de grado 1, con el cual se obtiene la propagación de onda sonora discreto [5] cuya variable dependiente es la función presión, $p$, se hace un mallado regular con triangularización $\tau_{h}$, de $m$ elementos finitos con longitud de lado $h$, representados por ${ }^{e}$, que se encuentra ubicados en el plano coordenado $\mathrm{XZ}$ como se muestra en la Fig. 1, cuyos nodos tienen como coordenadas $\left(x_{1}, z_{1}\right),\left(x_{2}, z_{2}\right)$ y $\left(x_{3}, z_{3}\right)$ las cuales son conocidas.

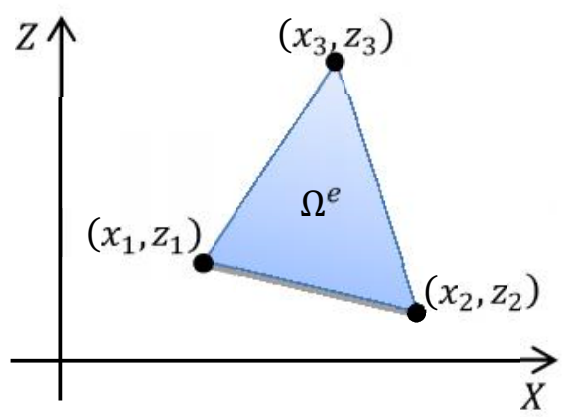

Fig. 1 Elemento de la triangulación $\tau_{h}$.

La aproximación consiste en expresar a $\bar{p}_{n}$ donde $\mathrm{m}$ es el número de nodos por elemento y sobre cada elemento $\varsigma^{e}, p^{e}$ es expresado por:

$$
p^{e}=\psi_{1}^{e} p_{1}^{e}+\psi_{2}^{e} p_{2}^{e}+\psi_{3}^{e} p_{3}^{e}
$$

Las funciones $\psi_{i}^{e}$ son las funciones de extrapolación dadas por:

$$
\begin{aligned}
\psi_{1}^{e} & =\frac{1}{2 A^{e}}\left[x_{2} z_{3}-x_{3} z_{2}+\left(z_{2}-z_{3}\right) x+\left(x_{3}-x_{2}\right) z\right] \\
\psi_{2}^{e} & =\frac{1}{2 A^{e}}\left[x_{3} z_{1}-x_{1} z_{3}+\left(z_{3}-z_{1}\right) x+\left(x_{1}-x_{3}\right) z\right] \\
\psi_{3}^{e} & =\frac{1}{2 A^{e}}\left[x_{1} z_{2}-x_{2} z_{1}+\left(z_{1}-z_{2}\right) x+\left(x_{2}-x_{1}\right) z\right]
\end{aligned}
$$

donde: $A^{e}$ representa el área del elemento triangular Fig. 1. Estas funciones tienen las siguientes propiedades:

$$
\sum_{i=1}^{3} \psi_{i}^{e}=1 \mathrm{y} \psi_{i}^{e}\left(x_{i}, z_{j}\right)=\delta_{i j}
$$

donde $\delta_{i j}$ es la función delta de Kronecker.

Los $p_{i}^{e}$ son funciones que dependen del tiempo $(t) \mathrm{y}$ de los nodos $\left(x_{i}, z_{i}\right) \mathrm{i}=1,2,3$, del elemento Fig.1.

Reemplazando en la ecuación (10) y sus respectivas derivadas de la ecuación (8), se obtiene el sistema matricial:

$$
M^{e} P_{e}^{\prime \prime}+K^{e} P_{e}=0
$$


Donde $M^{e}$ y $K^{e}$ son matrices y cuyos coeficientes son expresados por:

$$
\left\{\begin{array}{c}
M_{i j}^{e}=\frac{1}{c^{2}} \int \psi_{i} \psi_{j} d x d z \\
K_{i j}^{e}=\int\left(\frac{\partial \psi_{i}}{\partial x} \frac{\partial \psi_{j}}{\partial x}+\frac{\partial \psi_{i}}{\partial z} \frac{\partial \psi_{j}}{\partial z}\right) d x d z
\end{array}\right.
$$

$i, j=\{1,2,3\} . P_{e}^{\prime \prime}$ у $P_{e}$ son los vectores

$P_{e}=\left[p_{1}^{e} p_{2}^{e} p_{3}^{e}\right]^{T}$, denotando sus derivada respecto a $\mathrm{t}$, de cada componente por

$$
P_{e}^{\prime \prime}=\left[\frac{d^{2} p_{1}^{e}}{d t^{2}} \frac{d^{2} p_{2}^{e}}{d t^{2}} \frac{d^{2} p_{3}^{e}}{d t^{2}}\right]^{T}
$$

Luego de calcular cada una de las integrales sobre cada área de los elementos triangulares, se acopla éstas soluciones parciales y se obtiene un sistema de Ecuaciones Diferenciales Ordinarias (EDO) de segundo orden:

$$
M P_{h}{ }^{\prime \prime}+K P_{h}=0
$$

$M_{i j}=\sum_{e=1}^{m} M_{i j}^{e}$ y $K_{i j}=\sum_{e=1}^{m} K_{i j}^{e}$ y $P_{h}$ es el vector que contiene los valores nodales de la presión en el instante $t$ y en cada nodo de $\tau_{h}$.

Definiendo las condiciones del problema de valor inicial discreto (13) por:

$$
P_{h}(0)=G_{0} P_{h}{ }^{\prime}(0)=H_{0}
$$

Donde $G_{0}$ y $H_{0}$ son los vectores obtenidos de las condiciones iniciales dadas en (7). Ahora usando las aproximaciones dadas por el método de Newmark el sistema (13) con sus condiciones (14) se transforma en un sistema algebraico de ecuaciones lineales.

Haciendo $P_{s}=P_{h}(t s) \quad s=0, \ldots, N$. Se obtiene el sistema algebraico (15), donde:

$$
\begin{gathered}
\widehat{K} P_{s+1}=\hat{F}_{s} \\
\widehat{K}=K+a_{3} M \\
\hat{F}_{s}=M\left(a_{3} P_{s}+a_{4} P^{\prime}{ }_{s}+a_{5} P^{\prime \prime}{ }_{s}\right) \\
a_{3}=\frac{2}{\gamma(\Delta t)^{2}}, \quad a_{4}=\frac{2}{\gamma \Delta t} \quad, \quad a_{5}=\frac{1}{\gamma}-1,
\end{gathered}
$$

Algoritmo.-

Inicializando en $\mathrm{t}=\mathrm{t}_{0}$,

$$
P^{\prime \prime}{ }_{0}=-M^{-1} K P_{0}
$$

Calculando.-

$$
\begin{gathered}
\left\{\begin{array}{c}
P^{\prime \prime}{ }_{s+1}=a_{3}\left(P_{s+1}-P_{s}\right)-a_{4} P^{\prime}{ }_{s}-a_{5} P^{\prime \prime}{ }_{s} \\
P^{\prime}{ }_{s+1}=P^{\prime}{ }_{s}+a_{2} P^{\prime \prime}{ }_{s}+a_{1} P^{\prime \prime}{ }_{s+1}
\end{array}\right. \\
\text { con } a \_1=\alpha \Delta t \quad, \quad a \_2=(1-\alpha) \Delta t
\end{gathered}
$$

La convergencia de la solución de este sistema dependerá de los valores que se le dé a los parámetros $\alpha$ y $\gamma$.

Finalmente se resuelve el sistema algebraico (15), obteniéndose una solución aproximada $P_{S}$ del problema presión sonora en la etapa de tiempo $s$ en cada punto del mallado espacial almacenado en un vector $P$.

La convergencia del esquema el método Newmark [6] se mide con la norma de la energía definida por:

$$
\|v\|_{m}=\left(\sum_{|\alpha| \leq m} \int_{\Omega^{e}}\left|D^{\alpha} v\right|^{2} d \Omega\right)^{\frac{1}{2}}
$$

Así como para su estimación del error, $\left\|P-P_{s}\right\|_{m}$. La convergencia es del orden $\mathcal{O}\left(\Delta t^{l}\right)$, donde $l=2$, para $\alpha=\frac{1}{2} \mathrm{y} l=1$ para $\alpha>\frac{1}{2}$.

Una vez obtenido aproximadamente $P_{S}$, se puede calcular $P_{h}$, en el cual su estimación por el método de elementos finitos [ 3 y 7 ]], $\left\|P-P_{h}\right\|_{m}$ es del orden de $\mathcal{O}\left(\mathrm{h}^{\mathrm{l}}\right)$ donde $l=k+1-m$ y $k$ es el grado de los polinomios de aproximación $\psi_{i}$.

\section{RESULTADOS NUMERICOS}

Se toma una base de datos (Fig.4) experimental sobre una zona de estudio correspondiente al pabellón R1 de la Facultad de Ciencias, Fig. 2.

Expuesta a diferentes fuentes de ruido. 


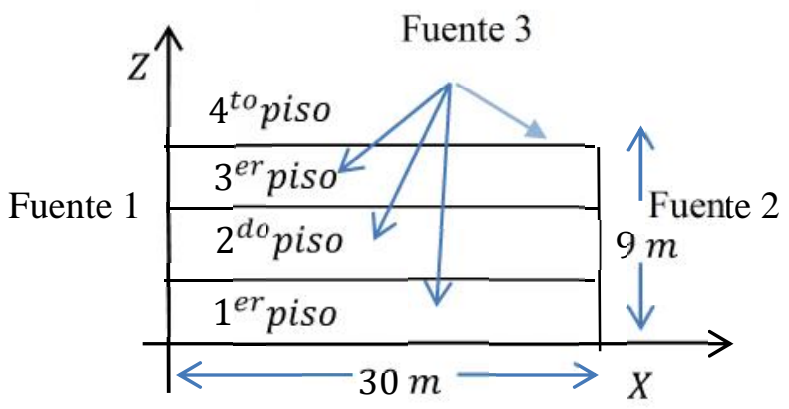

Fig. 2 Esquema del dominio .

Fuente de ruido 1.- Es vehicular de la Av. Túpac Amaru Fig. 3 y la construcción de la nueva Facultad de Ing. de Petróleo.

Fuente 2.- Aquí se encuentra el restaurante y la construcción de ampliación de la Facultad de Ciencias.

Fuente 3.- En estas zonas se considera el ruido de los peatones que circulan por los pasadizos.

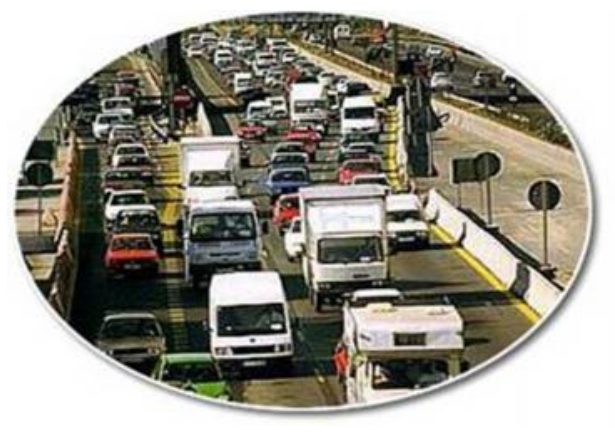

Fig. 3 Fuentes de ruido vehicular.

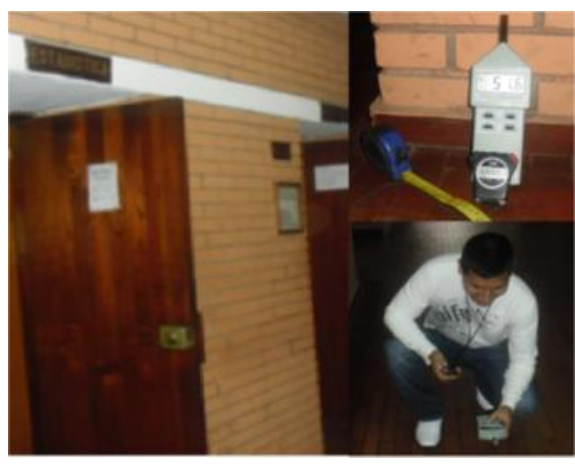

Fig. 4 Proceso de toma de datos.
Para la obtención de una condición inicial de presión numérica ésta es medida, ver Fig. 4, en un intervalo de tiempo [8:45 a.m. a 9:30 a.m] es decir, durante 45 minutos ya que sólo se dispone de un sonómetro.

Dichos datos se midieron a las 8:45 a.m.; luego se realiza una malla de 90 elementos Fig. 5 y se muestra la data experimental tomada en los nodos del mallado, ver Tabla 1 como condición inicial:

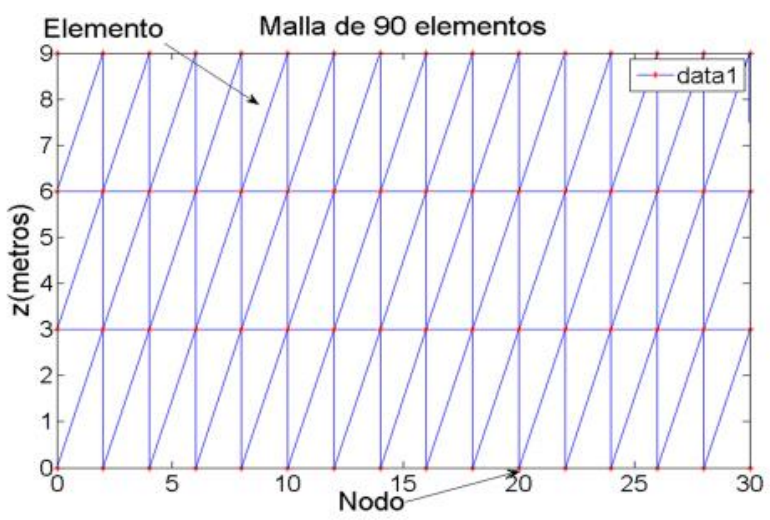

Fig. 5 Malla de 90 elementos triangulares.

Tabla 1. Condición inicial $(P[d B])$ sobre $\mathrm{z}_{\mathrm{j}}$ $\left(x_{i}, z_{j}\right)$ en metros.

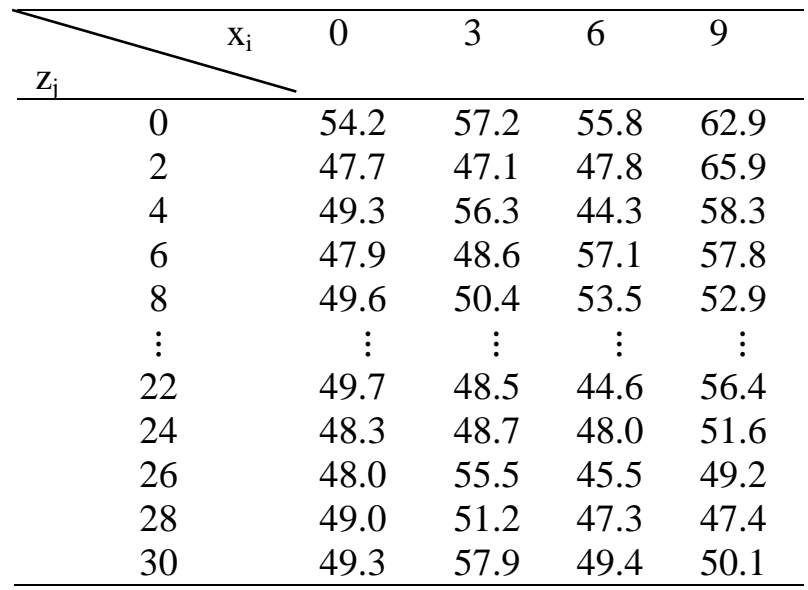

Además como se desea obtener más información se hace un promediado entre cada par de datos, obteniéndose así la presión inicial para 360 elementos Fig. 6 y cuya malla se muestra a continuación: 


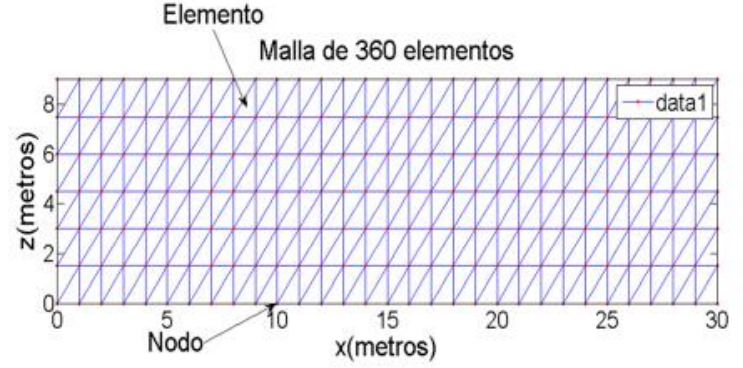

Fig. 6 Malla de 360 elementos triangulares.

Ahora como los cambios de presión varían muy rápidamente en el sonómetro no calibrado, su variación en tiempo es muy pequeña, por tanto se ha considerado que esta condición tiende a cero.

Luego para obtener las condiciones de contorno del dominio computacional $\Omega$, se procede a medir en el mismo horario 8:45 a.m, el mismo intervalo de tiempo (5 min) a lo largo de un pasadizo, ver Fig. 4 y se toma como $\Delta t=5 \mathrm{~min}$, luego se hace 8 mediciones más, tardándose un total de $45 \mathrm{~min}$, éste intervalo de tiempo es el que se considera en todo el proceso.

Tabla 2. Condición de contorno $1(P[d B])$ del lado izquierdo de cada 5 minutos.

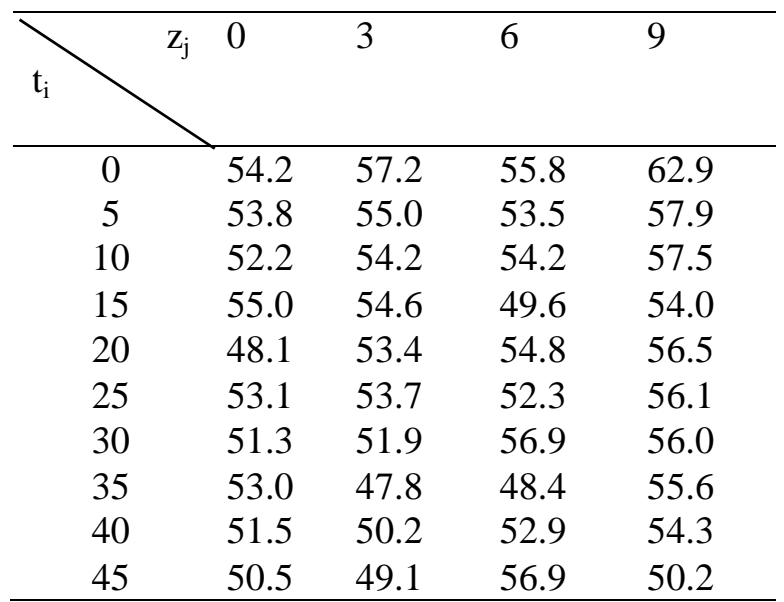

Tabla 3. Condición de contorno $2(P[d B])$ del lado derecho de cada 5 minutos.

\begin{tabular}{|c|c|c|c|c|}
\hline$z_{2}$ & 0 & 3 & 6 & 9 \\
\hline 0 & 49.3 & 57.9 & 49.4 & 50.1 \\
\hline 5 & 63.2 & 49.0 & 48.6 & 54.4 \\
\hline 10 & 62.7 & 57.2 & 48.8 & 49.4 \\
\hline 15 & 62.9 & 55.5 & 52.9 & 46.6 \\
\hline 20 & 58.2 & 49.9 & 64.3 & 46.7 \\
\hline 25 & 64.3 & 53.6 & 66.4 & 48.5 \\
\hline 30 & 57.7 & 49.5 & 67.5 & 48.0 \\
\hline 35 & 59.7 & 53.7 & 48.3 & 48.3 \\
\hline 40 & 62.2 & 48.6 & 44.3 & 56.9 \\
\hline 45 & 66.6 & 50.0 & 44.9 & 52.3 \\
\hline
\end{tabular}

Tabla 4. Condición de contorno $3(P[d B])$ del lado inferior de cada 5 minutos.

\begin{tabular}{|c|c|c|c|c|c|}
\hline & $t_{j}$ & 0 & 15 & 30 & 45 \\
\hline$x_{i}$ & & & & & \\
\hline 0 & & 54.2 & 58.6 & 47.1 & 50.0 \\
\hline 2 & & 47.7 & 56.5 & 45.1 & 47.1 \\
\hline 4 & & 49.3 & 55.0 & 43.1 & 48.6 \\
\hline 6 & & 47.9 & 48.1 & 46.8 & 47.5 \\
\hline 8 & & 49.6 & 59.5 & 48.7 & 45.2 \\
\hline & $\vdots$ & $\vdots$ & $\vdots$ & $\vdots$ & $\vdots$ \\
\hline 22 & & 49.7 & 50.8 & 48.1 & 45.0 \\
\hline 24 & & 48.3 & 67.2 & 50.1 & 58.0 \\
\hline 26 & & 48.0 & 68.5 & 53.3 & 45.1 \\
\hline 28 & & 49.0 & 67.2 & 51.2 & 48.0 \\
\hline 30 & & 49.3 & 73.2 & 55.8 & 50.7 \\
\hline
\end{tabular}

Tabla 5. Condición de contorno $4(P[d B])$ del lado superior de cada 5 minutos.

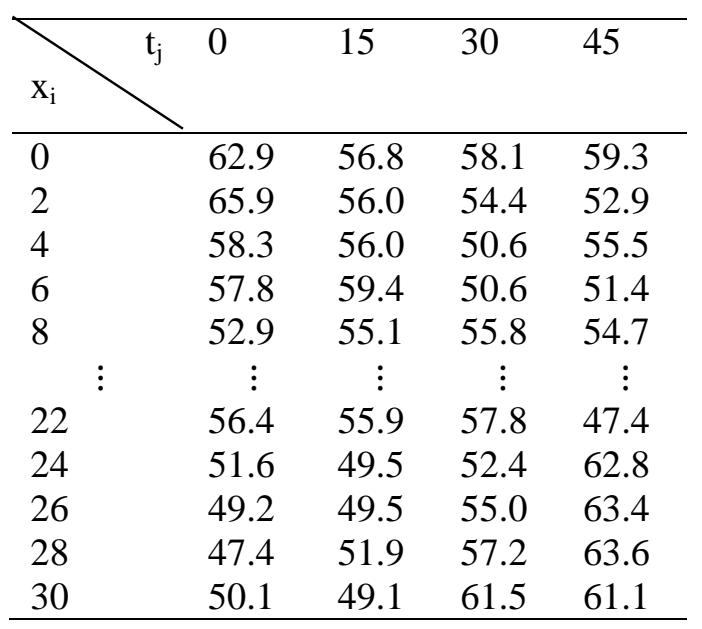

TECNIA 22 (2) 2012 
Ahora se muestra la data experimental de los cuatro contornos: lado izquierdo Tab.2, inferior Tab. 3, derecho Tab. 4 y superior Tab. 5. Luego se procede a calcular los diferentes niveles de presión en cada instante de tiempo, Figuras 7, 8, 9, 10, 11, 14, 15 y 16.

En el programa de cálculo construido para el algoritmo de solución del problema de presión sonora bidimensional, la convergencia se ha obtenido para los parámetros de estabilidad de Newmark $\alpha=0.5$ y $\gamma=0.5$.

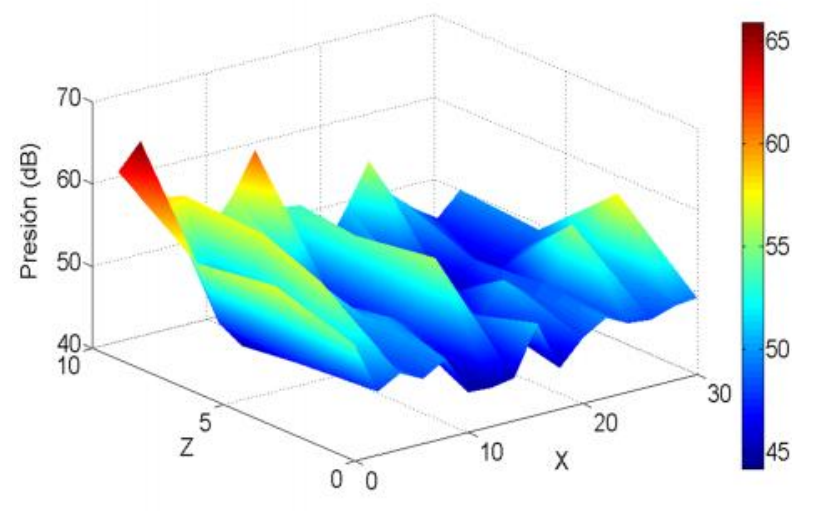

Fig. 7 Niveles de presión sonoro experimental o inicial para 90 elementos.

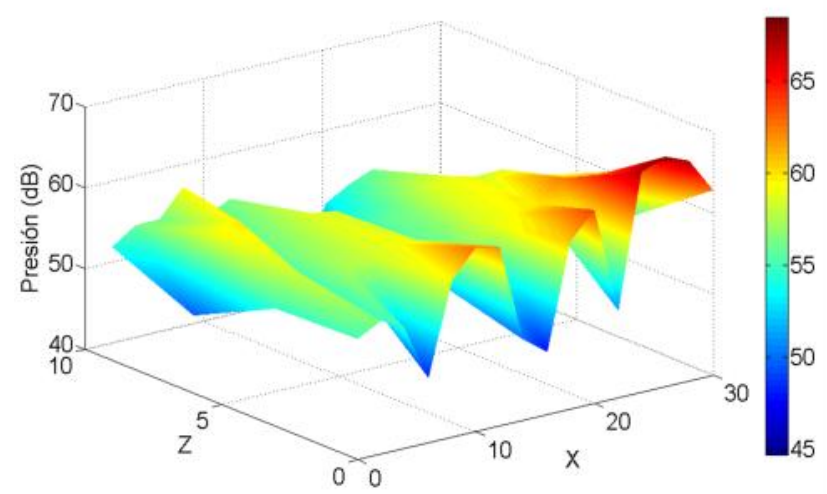

Fig. 8 Niveles de presión sonoro después de $15 \mathrm{~min}$ para 90 elementos.

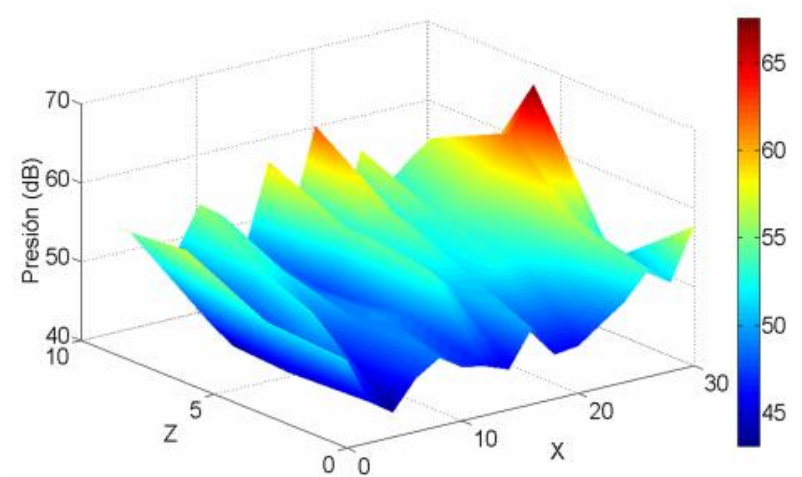

Fig. 9 Niveles de presión sonoro después de $30 \mathrm{~min}$ para 90 elementos.

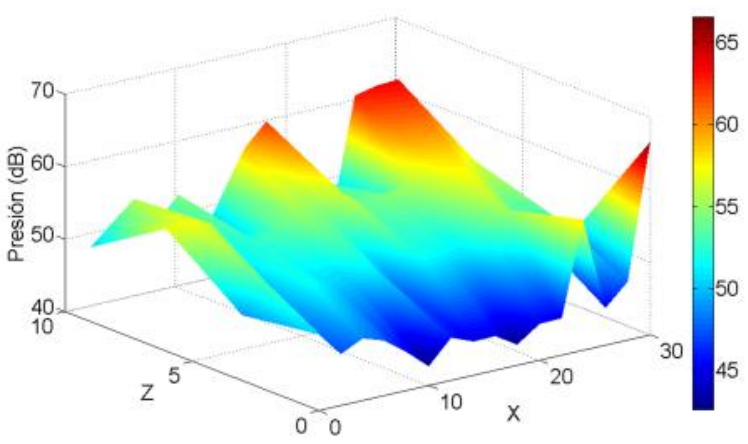

Fig. 10 Niveles de presión sonoro después de 45 min. para 90 elementos finitos.

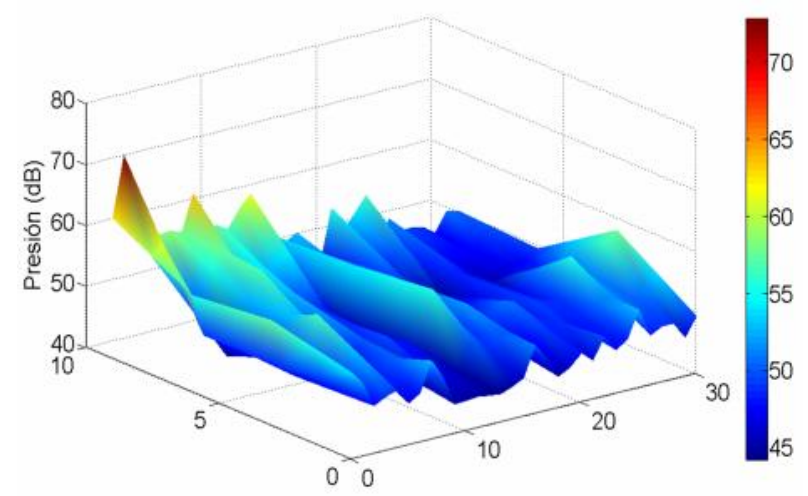

Fig.11 Niveles de presión sonoro experimental o inicial para 360 elementos. 


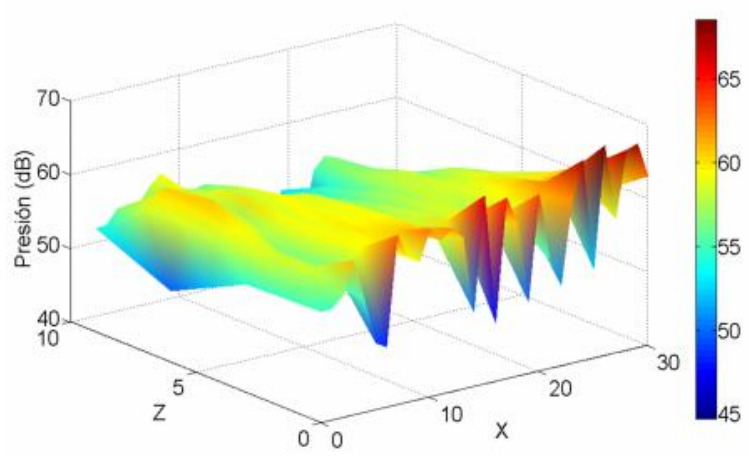

Fig. 12 Niveles de presión sonoro después de $15 \mathrm{~min}$ para 360 elementos finitos.

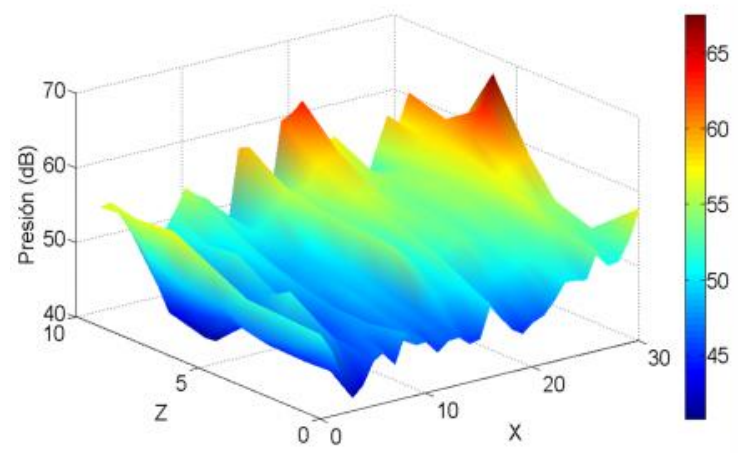

Fig. 13 Niveles de presión sonoro después de 30 min para 360 elementos.

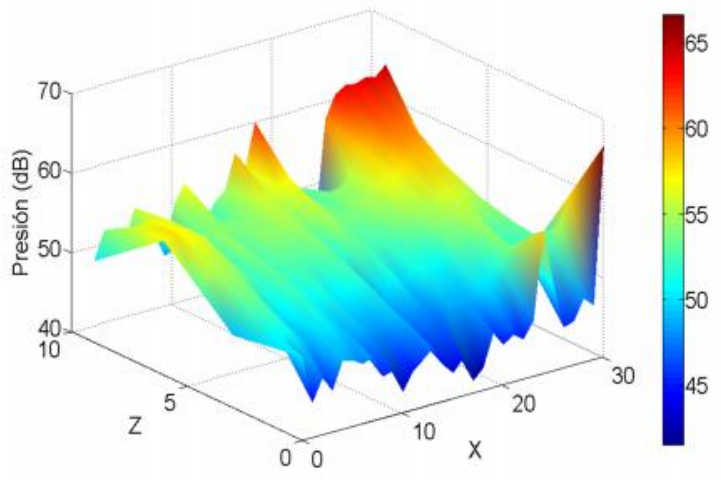

Fig.14 Niveles de presión sonoro después de $45 \mathrm{~min}$ para 360 elementos finitos.

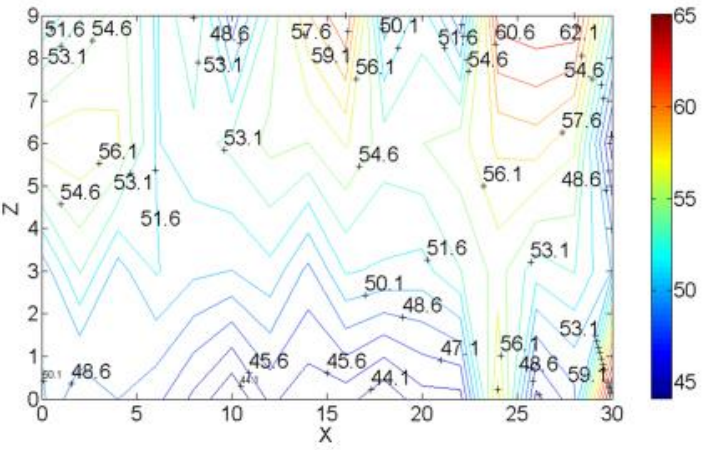

Fig. 15 Mapa de ruido después de 45 min para 90 elementos finitos.

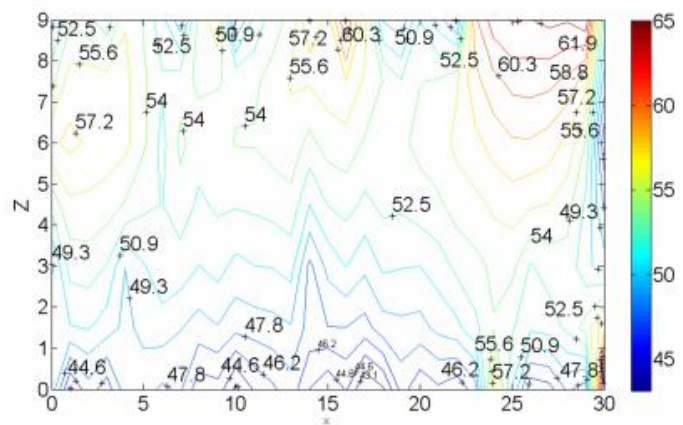

Fig. 16 Mapa de ruido después de 45 min para 360 elementos finitos.

\section{CONCLUSIONES}

Este trabajo contribuye al conocimiento de técnicas numéricas para construir de manera sencilla y económica un Mapa de Ruido, con un sólo Sonómetro.

La innovación es la construcción de la base de datos experimental para ser utilizada como condiciones iniciales, ver Tabla 1 y la medición de las condiciones de contorno (Tablas 2, 3, 4 y 5), para el desarrollo del problema de Presión Sonora.

La solución óptima se obtiene bajo la condición de convergencia basada en el método de Newmark, con el orden $\mathcal{O}\left(\Delta t^{l}\right)$, donde $l=2$, para $\alpha=\frac{1}{2}$ y $l=1$ para $\alpha>\frac{1}{2}$. Con estos últimos resultados se completa la obtención del Mapa de Ruido Figuras 12 y 13 del dominio computacional ubicado en el interior (aulas de la Facultad de Ciencias), donde se 
puede observar que se cumple la norma ambiental acústica.

Esta metodología de Elementos Finitos y Newmark se puede recomendar para el análisis de materiales acústico en 3D, optimizando los resultados con polinomios de interpolación de mayor grado, que se deja como problema abierto para futuros trabajos de investigación.

\section{AGRADECIMIENTOS}

Los autores agradecen al Instituto de Investigación de la Facultad de Ciencias, y al Doctor Humberto Asmat A, jefe de la Oficina Central de Planificación de la UNI, por todo el apoyo brindado al Laboratorio del Grupo LABOSIN de la UNI, donde se elaboró este trabajo.

\section{REFERENCIAS}

1. Möser Michael Barros, J. L., "Ingeniería Acústica, Teoría y Aplicaciones”, 2nda. Edición editorial Springer, 2009.
2. Tijonov, A., Samarsky, A., "Ecuaciones de la Física Matemática", 1era. edición editorial MIR, Moscú 1972.

3. Evans, Lawrence C., "Partial Differential Equations, Graduate Studies in Mathematics", Vol. 19, editorial American Mathematical Society, Berkeley 1997.

4. Quarteroni, A., "Numerical Mathematics", 2da. Edición, editorial Springer, 2007.

5. Reddy, J. N., "An Introduction to the Finite Element Method", 2nda. Edición editorial Mc Graw- Hill, 1993.

6. Chiba, F., Kako, T., "On the Stability Newmarks $\beta$-method". Research Institute of Mathematical Sciences, Kyoto University Vol. 1040, 39-44, 1998.

7. Mantilla, I, Munguia, J., "Método de D' Alambert con Integración de Simpson para resolver el problema de onda sonora", XV Encuentro de Física- FC-UNI, 2011.

Correspondencia: irlamn@uni.edu.pe

Recepción de originales: diciembre 2012

Aceptación de originales: marzo 2013 